\title{
Oxaliplatin/5-Fluorouracil/Leucovorin (FOLFOX4) Regimen as an Adjuvant Chemotherapy in the Treatment of Advanced Jejunal Adenocarcinoma: A Report of 2 Cases
}

\author{
Chao-Wen Chen ${ }^{a}$ Wen-Ming Wang ${ }^{b, d}$ Yu-Chung Su, deng-Yih Wu ${ }^{b}$ \\ Jan-Sing Hsieh ${ }^{c, d}$ Jaw-Yuan Wang ${ }^{c, d}$
}

Departments of a Emergency Medicine, ${ }^{\mathrm{b}}$ Internal Medicine and ${ }^{\mathrm{c} S u r g e r y, ~ K a o h s i u n g ~ M e d i c a l ~ U n i v e r s i t y ~ H o s p i t a l, ~}$ and ${ }^{d}$ Faculty of Medicine, College of Medicine, Kaohsiung Medical University, Kaohsiung, Taiwan

\section{Key Words}

FOLFOX4 • Adjuvant chemotherapy · Jejunum •

Adenocarcinoma

\begin{abstract}
Objective: To present our clinical experience of 5-fluorouracil/leucovorin/oxaliplatin (FOLFOX4) regimen administered as an adjuvant chemotherapy to 2 patients with advanced jejunal adenocarcinoma. Case Presentation and Intervention: A 55-year-old woman presented with recurrent upper abdominal pain, nausea and vomiting. A small bowel series as well as the abdominal computed tomography scan revealed an irregular narrowing lesion at the proximal jejunum. The patient then underwent an exploratory laparotomy and the jejunal adenocarcinoma with localized peritoneal metastasis was found (R0 resection, T3N1M1, stage IV). Chemotherapy with FOLFOX 4 regimen of 12 cycles was initiated after the curative resection. No adverse event was observed during the period of chemotherapy. She has been well without evidence of recurrence for over 20 months postoperatively. The second case was a 77-year-old female presenting with mechanical ileus. Surgical exploration revealed a proximal jejunal adenocarcinoma with regional lymph node involvement (R0 resection, T3N1M0, stage III). She also received the FOLFOX 4 chemotherapy of 12 cycles
\end{abstract}

with an uneventful course. No obvious toxicity developed except for temporary grade I peripheral neuropathy and skin eruption. This patient has survived well and has been free of this disease for over 12 months since the operation. Conclusion: This report showed that adjuvant chemotherapy with FOLFOX4 regimen seems effective and well tolerated in these 2 patients with advanced jejunal adenocarcinoma. Further investigation of a large number of patients with long-term follow-up is needed to confirm these findings.

Copyright $\odot 2008$ S. Karger AG, Basel

\section{Introduction}

Primary adenocarcinoma of the small bowel is a rare gastrointestinal malignancy, and although it is the most common type of small bowel cancer, its prevalence has been estimated between 0.2 and 0.6 per 100,000 in the overall population $[1,2]$. Until the present time, this disease remains a challenging problem for diagnosis and effective treatment. Early accurate diagnosis has been difficult because of its rarity, lack of specificity of clinical symptoms, and the limited diagnostic modalities, thereby leading to the frequently encountered advanced stages of the disease at presentation. Even with radical resection, recurrence/metastasis is common and prognosis is dis-

\section{KARGER}

Fax +41613061234

E-Mail karger@karger.ch

www.karger.com (c) 2008 S. Karger AG, Basel

1011-7571/08/0176-0496\$24.50/0

Accessible online at:

www.karger.com/mpp
Prof. Jaw-Yuan Wang, MD, PhD

Department of Surgery, Faculty of Medicine, College of Medicine

Kaohsiung Medical University, 100 Tzyou 1st Road

Kaohsiung 807 (Taiwan)

Tel. +886 7312 2805, Fax +886 7311 4679, E-Mail cy614112@ms14.hinet.net 
mal. When a small bowel adenocarcinoma has spread to other organs or the peritoneum, chemotherapy may be the only therapeutic option, although response rates to palliative combination chemotherapy are still low, and the median duration of survival is less than 1 year $[2,3]$.

Due to the rarity of this tumor, prospective evaluations of the efficacy of chemotherapy are infrequent, and most information is derived from descriptive studies that include retrospective analyses, case reports, and case series. Single- and multiple-agent regimens are empirically translated from experience with gastric and colorectal cancers. Combination chemotherapy with 5-fluorouracil (5-FU), folinic acid and oxaliplatin (FOLFOX4) has been shown to be synergistically efficacious in treating metastatic colorectal cancer [4], but little is known regarding the role of FOLFOX4 chemotherapy in advanced stages of the small bowel adenocarcinoma. Herein, we present the use of a FOLFOX4 regimen as an adjuvant chemotherapy administered to 2 patients with advanced jejunal adenocarcinoma (stages III and IV) after jejunal resection.

\section{Case Reports}

\section{Case 1}

A 55-year-old woman presented with complaints of recurrent upper abdominal pain, nausea and vomiting. Laboratory workup including blood routine, biochemistry, urinalysis, and stool routine uncovered nothing abnormal, except for mild anemia (hemoglobin $=10.5 \mathrm{~g} / \mathrm{dl}$ ). Upper gastrointestinal endoscopic survey was negative. After a few weeks of supportive management with oral medications for gastritis, her symptoms still persisted and progressed. A series of advanced examinations were then performed. A small bowel series disclosed an irregular narrowing lesion at the proximal jejunum with dilatation of upstream jejunum and duodenum (fig. 1). The subsequent abdominal computed tomography $(\mathrm{CT})$ scan revealed a focal annular wall thickening in the proximal jejunal loop. Based on the diagnosis of jejunal tumor, this patient then underwent a segmental resection of the small bowel with a $100-\mathrm{mm}$ margin at both ends. She also received the excision of mesentery and lymph nodes, as well as partial parietal peritonectomy, because localized parietal peritoneal seeding was found. Adenocarcinoma of the jejunum was then identified by the postoperative histological report. Tumor-free resection margin was achieved (R0 resection), but positive metastasis in lymph nodes and peritoneum was confirmed. She was finally diagnosed as T3N1M1, stage IV. After a few days of uneventful postoperative care, she was discharged. Due to her advanced illness, chemotherapy was initiated 3 weeks after the surgery. FOLFOX4 regimen was conducted, comprising a 2-hour infusion of $85 \mathrm{mg} / \mathrm{m}^{2}$ of oxaliplatin on day 1, a 2-hour infusion of leucovorin $200 \mathrm{mg} / \mathrm{m}^{2}$ concurrently with oxaliplatin on day 1 , followed by a bolus of 5 -FU $400 \mathrm{mg} / \mathrm{m}^{2}$, then continuous infusion of 5 -FU $600 \mathrm{mg} / \mathrm{m}^{2}$ over $22 \mathrm{~h}$ on days 1 and 2 . Courses were repeated every 2 weeks. Toxicity was assessed according to the National Cancer Institute's common toxicity criteria (version 3.0, http://ctep.cancer.gov/

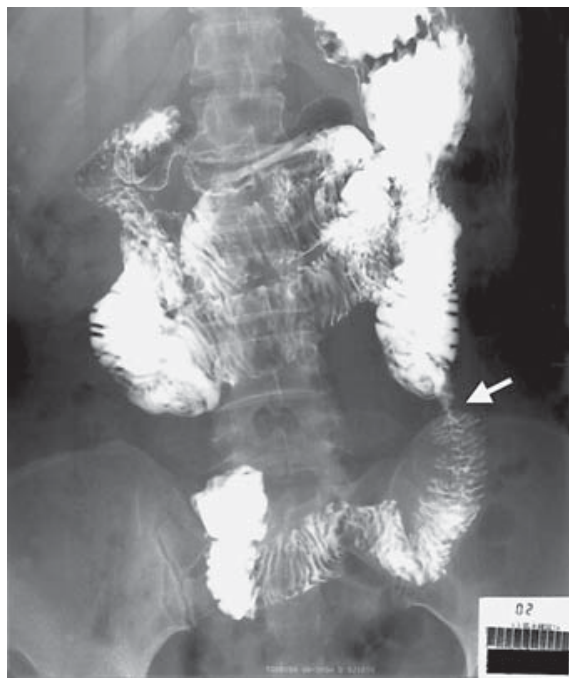

Fig. 1. The small bowel series reveals an irregular narrowing lesion at the proximal jejunum (arrow) with dilatation of upstream jejunum and duodenum.
forms/CTCAEv3.pdf) and no obvious adverse event was observed. The patient was carefully followed up regularly at 1-month intervals. At each visit, physical examination, routine blood workup, serum carcinoembryonic antigen measurement, liver function and renal function tests were conducted as appropriate. Chest X-ray and abdominal CT scan were performed every 6 months. Although accurate detection of peritoneal recurrence is always challenging in the posttreatment setting, no distant metastasis or local recurrence was identified with the traditional surveillance methods during her follow-up period. She has been free of disease for more than 20 months since the surgery.

Case 2

A 77-year-old woman was referred under the impression of mechanical ileus. She had a 5-day history of abdominal fullness, pain, nausea and vomiting. Physical examination revealed no fever or peritonism. Since no surgical abdomen was identified, she was admitted for detailed survey. Abdominal X-ray showed diffuse bowel gas and CT scan confirmed gross focal lumen narrowing at the proximal jejunum with neighboring bowel dilation (fig. 2). Diagnosis of jejunal tumor with mechanical ileus was made.

Exploratory laparotomy was done and revealed an annular thickened jejunal lesion about $7 \mathrm{~cm}$ in length, $10 \mathrm{~cm}$ distal to the ligament of Treitz. The involved bowel loop and adjacent mesentery were resected. Postoperative pathology revealed mucinous adenocarcinoma of jejunum. A proximal tumor-negative resection margin of $75 \mathrm{~mm}$ and a distal of $105 \mathrm{~mm}$ away from the lesion was achieved (R0 resection), but positive metastases in sampled lymph nodes were identified (T3N1M0, stage III). Her postoperative period was unremarkable, and she was discharged on her 16th postoperative day. After discharge, she was regularly followed up and received a total 12 cycles of FOLFOX 4 regimen as for the aforementioned case. No obvious toxicity developed except for 


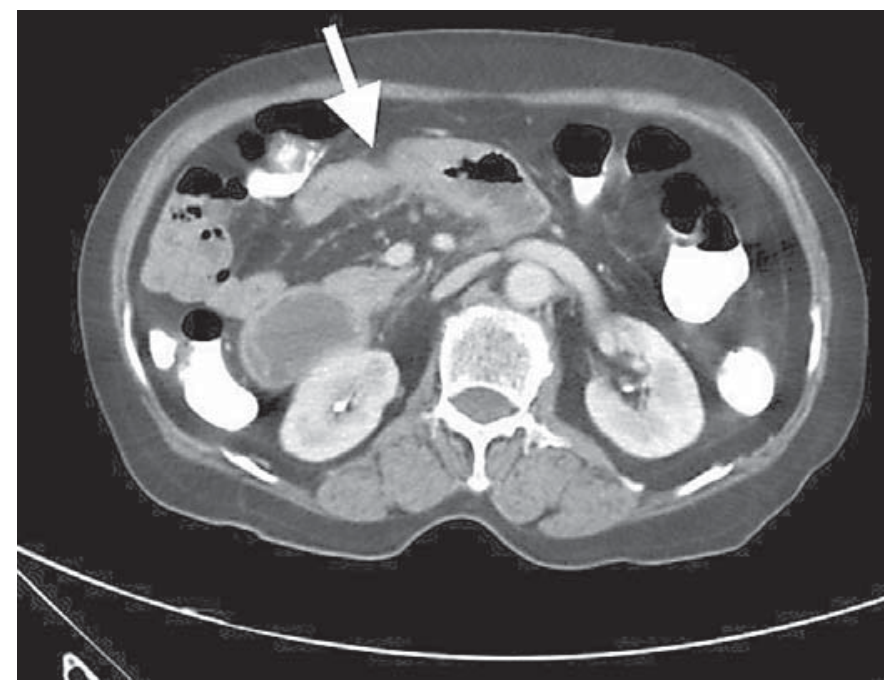

Fig. 2. Abdominal contrast-enhanced CT scan demonstrates focal lumen narrowing at the proximal jejunum with above-duodenal dilatation (arrow).

grade I peripheral neuropathy and skin eruption following chemotherapy. No dosage adjustment or treatment delay was performed. This symptom was mild and resolved gradually after the completion of chemotherapy. Follow-up CT scans showed no distant metastasis or local recurrence. The patient's physical status is fair now. She has survived well for over 12 months since abdominal surgery.

\section{Discussion}

Despite the fact that the small bowel occupies most of the length of the digestive system, related malignancies merely account for $1-3 \%$ of all gastrointestinal tract tumors [2]. Presenting symptoms and signs include a wide spectrum: most commonly pain, followed in prevalence by nausea and vomiting, anemia, weight loss, and gastrointestinal hemorrhage. These nonspecific features may occur in benign gastrointestinal conditions and usually conceal the actual malignancies. The rarity of this entity and its nonspecific symptoms result in a low index of suspicion. Most patients with small bowel cancer presented with advanced disease, and $74 \%$ of patients were categorized to stage III and IV [5].

Adenocarcinoma represents the most common type of small bowel cancer, and it usually occurs in the duodenum and jejunum. Radical surgery offers the best chance of a cure, although published outcomes for adenocarcinoma of the small bowel vary and depend on clinical stages [5, 6]. Dabaja et al. [5] reported a median overall survival time of 20 months, and a 5 -year overall survival rate of $26 \%$ [5], while Ugurlu et al. [6] reported a median survival time of 19 months, with $66 \%$ of patients who had a curative resection having tumor relapse. They also emphasized that tumor stage had a highly significant effect on median survival (72 months for stages I and II, 30 months for stage III, and 9 months for stage IV disease) [6]. With conventional follow-up methods including serum carcinoembryonic antigen monitoring, intensive utilization of abdominal CT or ultrasonography, and chest radiograph, detection of recurrence after surgery in small bowel cancer patients constitutes an important challenge. Improvement of modern imaging modalities such as multislice CT scan, MRI or PET scan has shown the potential to substitute conventional step-by-step or multimodality approaches, but a standard application has not been established.

Late identification of small bowel adenocarcinoma contributes to a dismal prognosis and chemotherapy is a common approach at such a stage. No clear recommendations for optimal treatment of small bowel cancer have been made although 5-FU and leucovorin are the most commonly used chemotherapy regimens for small bowel cancer $[7,8]$. Furthermore, combination therapy consisting of 5-FU, doxorubicin, and mitomycin C (the FAM regimen) [9] has been tried, but the results were not better than those of other chemotherapy combinations that include 5-FU and either cisplatin, carboplatin, or oxaliplatin [10]. L-Alanine, 6-thioguanine, Adriamycin, mitomycin or irinotecan etc. have also been reported [6, 8-10]. It is important to point out that these were uncontrolled studies with few patients, and hence no standard regimen was proved to be effective and accepted.

Oxaliplatin is a new third-generation platinum which is efficacious in treating advanced unresectable recurrent colorectal cancer and other solid tumors. When added to 5-FU and leucovorin, the regimen achieved a superior response rate and longer progression-free survival time compared with 5-FU/leucovorin alone as exemplified in the 'MOSAIC' trial. An improvement in disease-free survival for those with stage III disease for the oxaliplatin regimen compared to $5-\mathrm{FU} /$ leucovorin (68 vs. $58 \%$ ) was statistically significant $(\mathrm{p}<0.05)$ [11]. The combination of oxaliplatin with infusional 5-FU/leucovorin (FOLF$\mathrm{OX}$ ) is now established as the backbone of treatment for patients with metastatic colorectal cancer. Many clinicians accept 5-FU/leucovorin/oxaliplatin (FOLFOX) as a standard of care for patients with resected colon cancer with lymph node metastases [4]. 
The FOLFOX regimen has also been used for other gastrointestinal tract malignancies, like pancreatic cancers and gastric cancers. Within a tolerable level of toxicity, Nardi et al. [12] reported good tolerability of the FOLFOX4 regimen and a response rate of $32 \%$ in elderly patients with advanced gastric cancer. Recently, one investigation demonstrated that the FOLFOX4 regimen was effective in 3 patients with advanced small bowel adenocarcinoma. Likewise, our 2 patients received the FOLFOX4 regimen smoothly with an acceptable safety profile and favorable outcome. Their survival times after FOLFOX4 administration were at least over 14 and 6 months with an overall survival after primary diagnosis of at least over 20 and 12 months, respectively. Moreover, there were few adverse effects recorded within the followup period. Peripheral sensory neuropathy and skin eruption occurred in the second case but completely recovered after the completion of chemotherapy. Common side effects related to the FOLFOX regimen, such as neu- tropenia, thrombocytopenia, nausea, vomiting, and diarrhea, were not subjectively complained about nor objectively recognized.

\section{Conclusion}

This case shows that combination chemotherapy with the FOLFOX4 regimen as adjuvant treatment seems effective and well-tolerated in our patients with advanced jejunal adenocarcinoma. Further large-scale controlled trials are needed to confirm these findings.

\section{Acknowledgment}

We sincerely thank Dr. Ming-Chin Paul Shih and his colleagues for their excellent help with the preparation of the manuscript from the radiologist's point of view.

\section{References}

1 Barclay THC, Schapira DV: Malignant tumors of the small intestine. Cancer 1983;51: 878-881.

- 2 Kummar S, Ciesielski TE, Fogarasi MC: Management of small bowel adenocarcinoma. Oncology (Williston Park) 2002;16: 1364-1369; discussion 1370, 1372-1373.

-3 Neugut AI, Marvin MR, Rella VA, Chabot JA: An overview of adenocarcinoma of the small intestine. Oncology (Williston Park) 1997;11:529-536; discussion 545, 549-550.

4 Leichman CG: Adjuvant therapy for colon cancer 2005: new options in the twenty-first century. Surg Oncol Clin N Am 2006; 15: 159-173.

5 Dabaja BS, Suki D, Pro B, Bonnen M, Ajani $\mathrm{J}$ : Adenocarcinoma of the small bowel: presentation, prognostic factors, and outcome of 217 patients. Cancer 2004;101:518-526.
6 Ugurlu MM, Asoglu O, Potter DD, Barnes SA, Harmsen WS, Donobue JH: Adenocarcinomas of the jejunum and ileum: a 25 -year experience. J Gastrointest Surg 2005;9:11821188.

7 Ouriel K, Adams JT: Adenocarcinoma of the small intestine. Am J Surg 1984;147:66-71.

$\checkmark 8$ Crawley C, Ross P, Norman A, Hill A, Cunningham D: The Royal Marsden experience of small bowel adenocarcinoma treated with 5-fluorouracil. Br J Cancer 1998; 78:508510.

-9 Gibson MK, Holcroft CA, Kvols LK, Haller D: Phase II study of 5-fluorouracil, doxorubicin, and mitomycin $\mathrm{C}$ for metastatic small bowel adenocarcinoma. Oncologist 2005; 10: 132-137.
10 Locher C, Malka D, Boige V, Lebray P, Elias D, Lasser P, Ducreux M: Combination chemotherapy in advanced small bowel adenocarcinoma. Oncology 2005;69:290-294.

11 DeGramont A, Banzi M, Navarro M, Tabernero J, Hickish T, Bridgewater J, Rivera F, Figer A, Fountzilas G, Andre T: Oxaliplatin/5-FU/LV in adjuvant colon cancer: results of the international randomized MOSAIC trial (abstract 1015). Proc Am Soc Clin Oncol 2003;22:253a.

-12 Nardi M, Azzarello D, Maisano R, Del Medico P, Giannicola R, Raffaele M, Zavettieri M, Costarella S, Falzea A: FOLFOX-4 regimen as first-line chemotherapy in elderly patients with advanced gastric cancer: a safety study. J Chemother 2007;19:85-89. 